\title{
Simultaneous Determination of Multiple Polypeptide Antibiotics Residues in Lake Water by Lyophilization Combined with Liquid Chromatography-Tandem Mass Spectrometry
}

\author{
Jingli QIU,* Renping XIOng, ${ }^{*}$ Xuqin Song, ${ }^{* *}$ Meiyu Zhang, ${ }^{* *}$ Kanlin Peng, ${ }^{* *}$ Rong LIU, ${ }^{* * *}$ and \\ Limin $\mathrm{HE}^{* *}$ \\ *National Reference Laboratory of Veterinary Drug Residues (SCAU), College of Veterinary Medicine, South \\ China Agricultural University, Guangzhou, China \\ **Guangdong Provincial Key Laboratory of Veterinary Pharmaceutics, Development and Safety Evaluation, \\ South China Agricultural University, Guangzhou, China \\ ***Quality Supervision, Inspection and Testing Center for Domestic Animal Products, Ministry of Agriculture \\ and Rural Affairs, Guangzhou, China
}

\begin{abstract}
It is significant to develop a method for the simultaneous determination of multiple polypeptide antibiotics residues in lake water because of the emergence of multidrug-resistant microorganisms in water. A sensitive, eco-friendly and simple method was developed for the determination of multiple polypeptide antibiotics, including vancomycin, teicoplanin, polymyxin B, colistin and bacitracin A in lake water using liquid chromatography-tandem mass spectrometry (LC-MS/ MS). Water samples were lyophilized to enrich them after adjusting the $\mathrm{pH}$ to 3 . Then, $80 \%$ methanol in water containing $0.1 \%$ formic acid was used to reconstitute the residues for LC-MS/MS analysis. The results showed that target compounds were well separated and detected under the optimum instrumental conditions. The limits of detection and the limits of quantification of polypeptide antibiotics were in the range of $0.01-0.1$ and $0.02-0.2 \mathrm{ng} \mathrm{mL}^{-1}$, respectively. The matrixmatched calibration curves of all compounds were linear in the calibration range of $1-200 \mathrm{ng} \mathrm{mL}^{-1}$. At three spiked levels of $0.2(0.04), 0.4(0.1)$ and $1.0(0.2) \mathrm{ng} \mathrm{mL}^{-1}$ in lake water, the average recoveries of analytes were higher than $70 \%$, except for teicoplanin, with relative standard deviations of less than $20 \%$. Compared with other common sample pretreatment methods, the lyophilization process is simpler and more eco-friendly, achieving the simultaneous detection of multiple polypeptide antibiotics in lake water. The developed method is successfully applied to the routine monitoring of polypeptide antibiotics residues in lake water.
\end{abstract}

Keywords Polypeptide antibiotics, lyophilization, liquid chromatography-tandem mass spectrometry, lake water

(Received January 25, 2021; Accepted May 11, 2021; Advance Publication Released Online by J-STAGE May 21, 2021)

\section{Introduction}

Polypeptide antibiotics characterized with polypeptide structural are extracted from culture solutions of Actinomycetes, Bacillus, Streptomyces and so on. They are divided into three major classes, including glycopolypeptides (vancomycin, teicoplanin and avoparcin), lipolypeptides (daptomycin, polymyxin B (B1 and B2) and colistin (A and B)), and other polypeptides (bacitracin A), etc. ${ }^{1,2}$ These polypeptide antibiotics have a narrow antibacterial spectrum and are very active against Grampositive bacteria and Gram-negative bacteria. They are widely used in animal husbandry for a good effect on therapeutic respiratory tract infections, bovine mastitis as well as growthpromoting. ${ }^{2,3}$ At present, with an increasing emergence of multi-drug resistant (MDR) bacteria, ${ }^{4,5}$ some antibiotics have been banned as animal growth promoters, or even therapeutic medicine in some countries. ${ }^{6-9}$ Even though most polypeptide antibiotics have been banned, there were still cases of drug

$\dagger$ To whom correspondence should be addressed.

E-mail: 1ljrr@scau.edu.cn abuse. Due to the poor absorption of some polypeptide antibiotics by animals, they would be mostly excreted unchanged through feces and urine. ${ }^{10-12}$ The feces and urine could be applied to soil as a fertilizer, resulting in the accumulation and pollution of antibacterial drugs in soil and environmental water. Moreover, wastewater from livestock farms without the complete removal of antibiotics was directly discharged into lakes, rivers, and agricultural land, which was an important pollutant pathway for antibacterial drugs in the environment. ${ }^{13}$ It has been reported that antibacterial drugs in the environment could cause varying degrees of impact on organisms, which could lead to the increase of antibiotic-resistant bacteria and be hazardous to human health. ${ }^{10,14,15}$ Consequently, it is of great significance to establish a detection method to monitor multiple polypeptide antibiotics residues in lake water.

There are many reported methods concerning the determination of single or multiple polypeptide antibiotics residues in animal tissues and feed by using LC-MS/MS, 2,16,17 high-performance liquid chromatography (HPLC) combined with ultraviolet detector (UVD), ${ }^{18}$ fluorescence detector (FLD) ${ }^{19}$ and an evaporative light-scattering detection (ELSD) method. ${ }^{3}$ Since the concentration of antibiotics is very low in water, a highly 
sensitive detection instrument is beneficial to determine trace analytes. A LC-MS/MS analysis in a multi-reaction monitoring (MRM) mode is the preferred option.

Some analytical methods for single polypeptide in environmental water have been reported. Song et al. ${ }^{20}$ established a method to detect polymyxins in environmental water using HPLC-UV based on imprinted solid-phase extraction (SPE). The limits of detection (LODs) of polymyxins were between 1.0 and $2.0 \mathrm{ng} \mathrm{mL}^{-1}$. Li et al. ${ }^{21}$ and Rossmann et al. $^{22}$ developed methods to simultaneously detect vancomycin with other antibiotics in wastewater by LC-MS/MS based on SPE. The LODs of vancomycin were 25.7 and $73.5 \mathrm{ng} \mathrm{mL}^{-1}$, respectively. While there are some analytical methods that can detect colistin and vancomycin in environmental water, there is still no method for the simultaneous detection multiple polypeptide drugs in lake water. This may be due to the presence of multiple ions in lake water, which would seriously affect the interaction between the polypeptide drugs and the SPE column packing. Accordingly, the simultaneous enrichment of multiple polypeptide drugs in lake water by SPE is difficult to achieve. What is more, the SPE approach is time-consuming, including steps for sample loading, washing, and elution, and thus resulting in analytes' loss. Because of the different physical and chemical properties of multiple analytes, it is difficult to find an appropriate SPE cartridge, which can simultaneously retain compounds with diverse structures and properties for obtaining high recoveries. The lyophilization process, also known as freeze-drying, is commonly used in the preservation of food, biologicals, and pharmaceutical materials..$^{23}$ It is also used in sample preparation to simplify the extraction process for drug residue analysis. ${ }^{24-28}$ This pretreatment method has the advantages of simplicity, less drug loss and less use of organic solvents. Hu et al. reported a method for the LC-MS/MS determination of 26 veterinary analytes in different environmental water matrices, including groundwater, fish pond water, biogas digester water, and lagoon wastewater. Water samples were enriched by 50 times by lyophilization and the LOD was low (range from 0.1 to $6.5 \mathrm{ng} \mathrm{L}^{-1}$ ). ${ }^{26}$ Hirsch et al. established an LC-MS/MS method for detecting 18 antibiotics in water samples via lyophilization. ${ }^{27}$ Qiu et al. developed a method to simultaneously determine ten aminoglycosides in four environmental water matrices by lyophilization combined with LC-MS/MS; the average recoveries of ten analytes ranged from 65.8 to $115.7 \%$ with relative standard deviations (RSDs) of less than $20 \% .28$

In this study, we first established a simple, sensitive and reliable LC-MS/MS method for the simultaneous detection of 9 common polypeptide antibiotics (vancomycin, teicoplanin A2-1, teicoplanin A2-2\&A2-3, teicoplanin A2-4\&A2-5, polymyxin B1, polymyxin B2, colistin A, colistin B and bacitracin A) residues in lake water. In order to obtain suitable recoveries and an ideal purifying effect, the LC-MS/MS analysis and lyophilization conditions were optimized, including using a chromatographic column, the mobile phase, the lyophilization $\mathrm{pH}$ and a reconstitution solution. The developed method was validated by the parameters of selectivity, LOD, limit of quantification (LOQ), linearity, recovery, precision and matrix effects (ME). Finally, it was successfully applied to the detection of actual lake water samples.

\section{Experimental}

Reagents and chemicals

Reference standards of vancomycin, teicoplanin (A2-1,
A2-2\&A2-3 and A2-4\&A2-5) and bacitracin A, were purchased from National Institutes for Food and Drug Control (Beijing, China). Polymyxin B (B1 and B2) was obtained from Dr. Ehrenstrofer GmbH (Augsburg, Germany). Colistin (A and B) was available from TRC (Toronto, Canada). The purity of all standards is higher than $88.6 \%$.

Individual stock solutions $(1 \mathrm{mg} \mathrm{mL}-1)$ were prepared by dissolving each compound in $0.1 \%$ formic acid (FA) in water and stored in the dark at $-20^{\circ} \mathrm{C}$ for 3 months. Mixed working solutions $\left(100 \mu \mathrm{g} \mathrm{mL}^{-1}\right)$ were prepared by mixing and diluting standard stock solutions with $0.1 \%$ FA in water fresh daily.

Methanol and acetonitrile (HPLC grade) were purchased from Fisher (Fair Lawn, NJ, USA). FA (HPLC grade) was obtained from Sigma-Aldrich (Munich, Germany) and hydrochloric acid (reagent grade) was supplied by the Guangzhou Chemical Reagent Company (Guangzhou, China). Ultrapure water was acquired by a Milli-Q Millipore system (Molsheim, France). Microporous membrane filters (1 and $0.22 \mu \mathrm{m}$ ) were bought from Jinteng Laboratory Equipment Co., Ltd. (Tianjin, China).

\section{Sample collection and pretreatment}

For the purposes of method development and validation, the lake water collected from South China Agricultural University was regarded as blank water samples. It was ananlyzed by LC-MS/MS, which showed that there were no target compounds in it. After collection, water samples were immediately filtered through $1 \mu \mathrm{m}$ microporous membrane filters, followed by $0.22 \mu \mathrm{m}$ microporous membrane filters to remove tiny suspended particles, and then divided into several portions and stored at $-20^{\circ} \mathrm{C}$ in the dark.

\section{Lyophilization and reconstitution}

Collected lake water was adjusted to $\mathrm{pH} 3$ with a $6 \mathrm{~mol} \mathrm{~L}^{-1}$ hydrochloric acid $(\mathrm{HCl})$ solution, and then aliquoted. Then $50 \mathrm{~mL}$ lake water was transferred into a $100 \mathrm{~mL}$ round-bottom centrifuge tube, and was spiked by appropriate working standard solutions to prepare fortified samples. These samples were completely frozen at $-80^{\circ} \mathrm{C}$ for $3 \mathrm{~h}$, and were then freeze-dried using an Alpha 2-4 LD plus lyophilizer (Christ, Germany) equipped with a core temperature of $-86^{\circ} \mathrm{C}$ and a vacuum of $0.1 \mathrm{mbar}$ for $24 \mathrm{~h}$.

After lyophilization, the residues were reconstituted in $1 \mathrm{~mL}$ of $0.1 \%$ FA in methanol- $0.1 \%$ FA in water $(80: 20, \mathrm{v} / \mathrm{v})$, and then it was sonicated for $10 \mathrm{~min}$ to dissolve the target compounds completely, and finally centrifuged at $15000 \mathrm{~g}$ for $10 \mathrm{~min}$ at $4{ }^{\circ} \mathrm{C}$ prior to LC-MS/MS.

\section{LC-MS/MS conditions}

A liquid chromatography system consists of a Shimadzu highperformance liquid chromatography system (Kyoto, Japan), equipped with a SIL-30AC autosampler, a LC-30AD binary pump, a CBM-20A controller and a CTO-20A thermostatic column oven (Kyoto, Japan). The separation of analytes was carried out on a Phenomenex Luna $\mathrm{C}_{18}$ column $(150 \mathrm{~mm} \times$ $2.1 \mathrm{~mm}$ i.d., $5 \mu \mathrm{m}$, Torrance, CA, USA) at $30^{\circ} \mathrm{C}$. A flow-rate of $0.2 \mathrm{~mL} \mathrm{~min}^{-1}$ and an injection volume of $10 \mu \mathrm{L}$ were used for analysis. The mobile phase consisted of $0.1 \%$ FA in acetonitrile (A) and $0.1 \% \mathrm{FA}$ in water (B). The gradient elution program was applied as follows: $0-2 \min , 6 \% \mathrm{~A} ; 2-5 \min , 6-40 \% \mathrm{~A}$; 5-14 min, $40-70 \% \quad \mathrm{~A} ; \quad 14-14.1 \mathrm{~min}, \quad 70-6 \% \quad \mathrm{~A}$; 14.1 - 18 min, 6\% A.

Mass spectrometry analyses were performed on an API 5500 triple quadruple mass spectrometer with an electrospray ionization (ESI) source and Analyst 1.6.2 software (Foster City, CA, USA). ESI was operated at the positive ionization mode. 
Table 1 MRM conditions for analytes in the positive ion mode ${ }^{a}$

\begin{tabular}{|c|c|c|c|c|c|c|}
\hline Compound & Ion & Precursor ion & Product ion ${ }^{\mathrm{b}}$ & $\mathrm{DP} / \mathrm{V}^{\mathrm{c}}$ & $\mathrm{CE} / \mathrm{eV}^{\mathrm{d}}$ & $\mathrm{RT} / \mathrm{min}^{\circ}$ \\
\hline \multirow[t]{2}{*}{ Vancomycin } & {$[\mathrm{M}+2 \mathrm{H}]^{2+}$} & 725.7 & $144.2 *$ & 105 & 25 & 6.74 \\
\hline & & & 99.9 & 100 & 60 & \\
\hline \multirow[t]{2}{*}{ Teicoplain A2-1 } & {$[\mathrm{M}+2 \mathrm{H}]^{2+}$} & 940.0 & $316.2 *$ & 65 & 25 & 7.67 \\
\hline & & & 204.4 & 65 & 35 & \\
\hline \multirow{2}{*}{ Teicoplain A2-2\&A2-3 } & {$[\mathrm{M}+2 \mathrm{H}]^{2+}$} & 941.0 & $316.4 *$ & 63 & 25 & 7.67 \\
\hline & & & 204.4 & 63 & 25 & \\
\hline \multirow{2}{*}{ Teicoplain A2-4\&A2-5 } & {$[\mathrm{M}+2 \mathrm{H}]^{2+}$} & 948.1 & $330.4 *$ & 60 & 27 & 7.85 \\
\hline & & & 204.5 & 60 & 35 & \\
\hline \multirow[t]{2}{*}{ Polymyxin B1 } & {$[\mathrm{M}+3 \mathrm{H}]^{3+}$} & 402.1 & $101.1^{*}$ & 65 & 23 & 7.02 \\
\hline & & & 396.6 & 65 & 19 & \\
\hline \multirow[t]{2}{*}{ Polymyxin B2 } & {$[\mathrm{M}+3 \mathrm{H}]^{3+}$} & 397.3 & $101.1^{*}$ & 70 & 16.5 & 6.95 \\
\hline & & & 391.7 & 70 & 23 & \\
\hline \multirow[t]{2}{*}{ Colistin A } & {$[\mathrm{M}+3 \mathrm{H}]^{3+}$} & 390.8 & $385.1^{*}$ & 70 & 23 & 6.98 \\
\hline & & & 101.1 & 70 & 19 & \\
\hline \multirow[t]{2}{*}{ Colistin B } & {$[\mathrm{M}+3 \mathrm{H}]^{3+}$} & 386.1 & $380.4^{*}$ & 75 & 30 & 6.89 \\
\hline & & & 101.1 & 75 & 23 & \\
\hline \multirow[t]{2}{*}{ Bacitracin A } & {$[\mathrm{M}+3 \mathrm{H}]^{3+}$} & 475.2 & $199.0^{*}$ & 72 & 43 & 7.39 \\
\hline & & & 227.3 & 70 & 35 & \\
\hline
\end{tabular}

a. MRM, multi-reaction monitoring. b. Product ion, the product ion $(*)$ of each analyte was used for quantification, and the other was used for identification. c. DP, declustering potential. d. CE, collision energy. e. RT, retention time.

The monitoring mode was MRM. Other conditions were operated as follows: ion spray voltage, $5500 \mathrm{~V}$; curtain gas, $40 \mathrm{psi}$; ion source temperature, $600^{\circ} \mathrm{C}$; auxiliary gas, $55 \mathrm{psi}$; and dwell time, $50 \mathrm{~ms}$, respectively. The mass spectral parameters of the target analytes are given in Table 1 .

\section{Method validation}

Validation of the developed method was performed by evaluating the selectivity, LOD, LOQ, linearity, recovery, precision, ME, etc.

The selectivity of the method was validated by analyzing 50 blank lake water samples from different lakes on the LC-MS/ MS. Blank samples were prepared under the lyophilization condition and followed by a reconstitution procedure. It is supportable when there is no interfering substance within the $2.5 \%$ margin of the retention time of the target analytes.

The LODs and LOQs were validated by analyzing a series of spiked water samples and calculated according to the concentration of the analytes when the signal-to-noise ratio $(S / N)$ is equal to or higher than 3 and 10 , respectively.

In order to compensate for the matrix effect, a matrix-matched calibration curve was used to test the linearity of the developed method. The reconstituted solution was used to dilute a mixed working standard solution to obtain the spiked standard concentrations of six points. When the coefficient of correlation $\left(r^{2}\right)$ is higher than 0.99 , the linearity of the method is acceptable.

The recoveries and precision were evaluated by spiking blank water samples at three concentration levels: low $(0.2(0.04)$

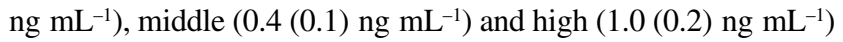
for target analytes in five replicates. The precision of intra-day and inter-day was expressed as the RSD. The intra-day RSD was determined for the same concentration within a day at three concentration levels in five replicates. The inter-day RSD was evaluated on three different days. The final concentration of the spiked sample was calculated by a matrix-matched standard curve. The recoveries of target analytes were calculated by the ratio of the concentration obtained from the matrix-matched calibration curve versus the corresponding concentration spiked in a blank sample.

In LC-MS/MS analysis, the complex matrix probably interferes with the analysis and determination of the target which is called ME. The ME of the sample is evaluated by calculating the peak areas of a matrix matched-standard solution with the corresponding standard working solution, expressing as the following equation:

$$
\operatorname{ME}(\%)=\left(\frac{B}{A}-1\right) \times 100
$$

where $A$ and $B$ represent the peak areas of the standard solution and the standard spiked after extraction, respectively. The blank lake water samples were enriched and purified according to the pretreatment method described, and then spiked corresponding standard solutions to prepare matrices standards. The value of $M E$ in the ranges of $0- \pm 20 \%, \pm 20-50 \%$ and $>50 \%$ (or $<-50 \%$ ) present the soft, medium and strong matrix effects, respectively.

\section{Results and Discussion}

\section{Optimization of MS/MS parameters}

Selecting the precursor and product ions was a key first step in developing this method. The individual standard solution of target analytes $\left(1 \mu \mathrm{g} \mathrm{mL}^{-1}\right)$ was in $50 \%$ methanol in water; they were then directly injected into the mass spectrometer with a syringe pump at a rate of $10 \mu \mathrm{L} \mathrm{min}^{-1}$. In the $\mathrm{ESI}^{+}$ion mode, the drugs would be more efficiently ionized, and had a higher sensitivity. Double-charge ions $\left([\mathrm{M}+2 \mathrm{H}]^{2+}\right)$ and triple-charge ions $\left([\mathrm{M}+3 \mathrm{H}]^{3+}\right)$ of target compounds were investigated. The results showed that the $[\mathrm{M}+3 \mathrm{H}]^{3+}$ of polymyxin $\mathrm{B}$, colistin, and bacitracin A have better ion responses. The responses of $[\mathrm{M}+2 \mathrm{H}]^{2+}$ for vancomycin and teicoplanin were stronger. These ions were used as precursor ions to produce product ions. Two product ions of each drug were screened out, in which a high response value indicates a quantitative ion, and the other is as a qualitative ion. This is in accordance with the requisition of the 2002/657/EC (IPs $\geq 4$ ). In the MRM mode, the remaining mass spectrometer parameters were optimized, mainly including the declustering potential (DP) and the collision energy (CE), and 
so forth. The optimized mass spectrometer parameters of the target analytes were performed as described in our previous publication, and shown in Table $1 .{ }^{17}$ It is noteworthy that teicoplanin A2-2\&A2-3 are a pair of isomers with the same mass spectrometry performance and retention time, as that of teicoplanin A2-4\&A2-5. In our study, this was measured without isomeric separation for teicoplanin.

\section{Optimization of chromatographic conditions}

In order to acquire a better peak shape and separation for the analytes, the chromatographic conditions were optimized. For the chromatographic column, Phenomenex Kinetex Biphenyl column $(50 \times 2.1 \mathrm{~mm}$ i.d., $2.6 \mu \mathrm{m})$, Phenomenex Luna $\mathrm{C}_{18}(150 \times$ $2.1 \mathrm{~mm}$ i.d., $5 \mu \mathrm{m})$ and Agilent Zorbax SB-C $18(150 \times 2.1 \mathrm{~mm}$ i.d., $3.5 \mu \mathrm{m})$ reversed-phase column were compared. The results indicated that the Phenomenex Luna $\mathrm{C}_{18}$ chromatographic column was selected as the analytical column because it gave good resolution, narrow peak, good symmetry, a better retention time stability and little peak tailing for all analytes.

The terminal amine groups of polypeptide compounds show a strong adsorption affinity with the stationary phase of the chromatographic column, causing significant peak tailing. For mobile phase, it is reported that if weakly acidic mobile phases were used, such undesirable interaction could be minimized. ${ }^{29}$ Methanol and acetonitrile were generally used as the organic phase in the mobile phase from the literature..$^{3,16-20}$ In this study, the results showed that when methanol was used, the separation effect of the target analytes was clearly inferior to acetonitrile. Consequently, $0.1 \% \mathrm{FA}$ in water and $0.1 \% \mathrm{FA}$ in acetonitrile were selected as the mobile phase. The gradient elution procedure was further optimized to obtain a better resolution and peak shape in a shorter time and to improve the sensitivity.

\section{Optimization of sample preparation}

The traditional extraction methods, such as SPE, are usually used to enrich drugs in water. For example, Song et al. ${ }^{20}$ synthesized molecularly imprinted polymers (MIP) for the selective extraction of polymyxins from environmental water samples, which could remove much more matrix interferences, reduce baseline noises significantly, and obtain a low LOD using an HPLC-UV detector. Li et al. ${ }^{21}$ and Rossmann et al. ${ }^{22}$ simultaneously detected vancomycin with other antibiotics in wastewater by LC-MS/MS based on SPE. However, there are some limitations in the process of synthesizing MIP and passing through the column, such as complication, tediousness as well as the waste of organic solvents. Lyophilization is eco-friendly with the use of less organic reagent, simple to operate and cost-effective. There were some published reports about lyophilization as an enrichment step to prepare water samples. ${ }^{26-28}$ In terms of lake water samples, lyophilization would be a good option for enriching polypeptide antibiotics.

During the lyophilization process, an appropriate lyophilization medium can allow the drug to be retained and enriched more effectively. Orwa et al. studied the stability of polymyxins in aqueous solution; the result showed that polymyxins could be more stable in acidic media while being more easily degraded when the $\mathrm{pH}$ value exceeded 5. Polymyxins are prone to degradation, and this degradation is faster with higher the $\mathrm{pH}$ values, generating different degradation products. ${ }^{30}$ The $\mathrm{pH}$ value has a great influence on polypeptide antibiotics in this process. In this part, different $\mathrm{pH}$ conditions (1,3,5 and 7) were optimized for obtaining higher recovery of target compounds from freeze-dried lake water. As shown in Fig. 1, the results demonstrated that under $\mathrm{pH}$ values of 1 and 3 , the recoveries of all drugs were higher than that of 5 and 7 .

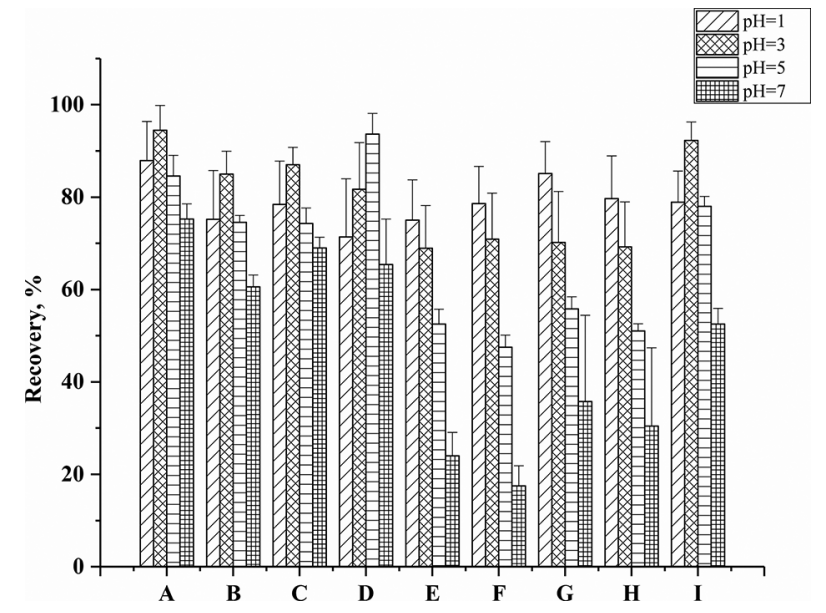

Fig. 1 Influence of various $\mathrm{pH}$ values on the recoveries of polypeptide antibiotics. A - I represent vancomycin, teicoplanin A2-1, teicoplanin A2-2\&A2-3, teicoplanin A2-4\&A2-5, polymyxin B1, polymyxin $\mathrm{B} 2$, colistin A, colistin B and bacitracin A, respectively.

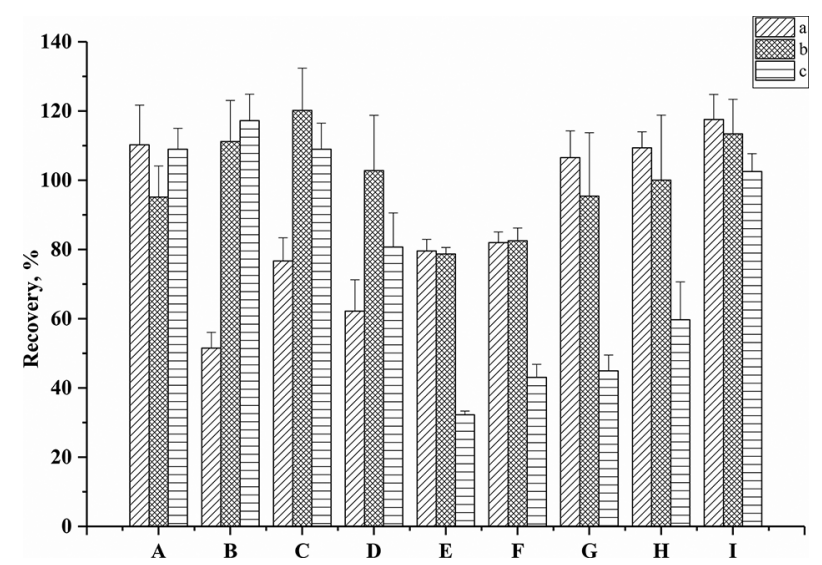

Fig. 2 Influence of various reconstitution solvents on the recoveries of polypeptide antibiotics. A - I represent vancomycin, teicoplanin A2-1, teicoplanin A2-2\&A2-3, teicoplanin A2-4\&A2-5, polymyxin $\mathrm{B} 1$, polymyxin B2, colistin A, colistin B and bacitracin A, respectively. a, $0.1 \%$ formic acid in methanol- $0.1 \%$ formic acid in water (100:0, $\mathrm{v} / \mathrm{v}) ; \mathrm{b}, 0.1 \%$ formic acid in methanol- $0.1 \%$ formic acid in water $(80: 20, \mathrm{v} / \mathrm{v})$; c, $0.1 \%$ formic acid in methanol-0.1\% formic acid in water $(50: 50, \mathrm{v} / \mathrm{v})$.

However, the lower was the $\mathrm{pH}$, and the more acid used, contributed to the worse environmental impacts. Accordingly, a $\mathrm{pH}$ value of 3 was selected as the final experimental freezedrying condition. After the water sample was lyophilized, the appropriate solution should be selected to fully dissolve the freeze-dried lake water sample matrices.

The response value and the recoveries of target drugs could be improved by the addition of FA in the reconstitution. Therefore, solutions of different proportion of $0.1 \%$ FA in methanol- $0.1 \%$ FA in water $(50: 50,80: 20$ and 100:0, v/v) were compared to reconstitute the lyophilized extracts of the water sample. The results are given in Fig. 2, which indicate that when using $0.1 \%$ FA in methanol-0.1\% FA in water $(80: 20, \mathrm{v} / \mathrm{v})$ as the reconstitution solution, the recoveries for all target compounds were simultaneously over $75 \%$. In addition, the increase in the proportion of methanol could diminish the dissolution of 


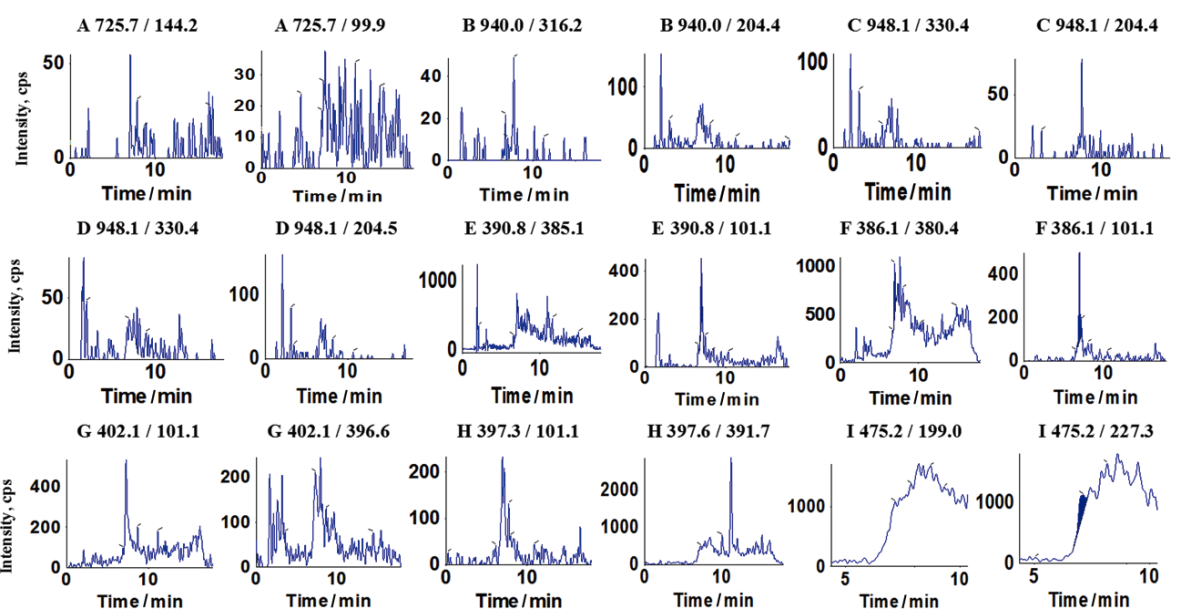

Fig. 3 Typical multi-reaction monitoring chromatogram of a blank lake water extract. A - I represent vancomycin, teicoplanin A2-1, teicoplanin A2-2\&A2-3, teicoplanin A2-4\&A2-5, polymyxin B1, polymyxin $\mathrm{B} 2$, colistin A, colistin B and bacitracin A, respectively.

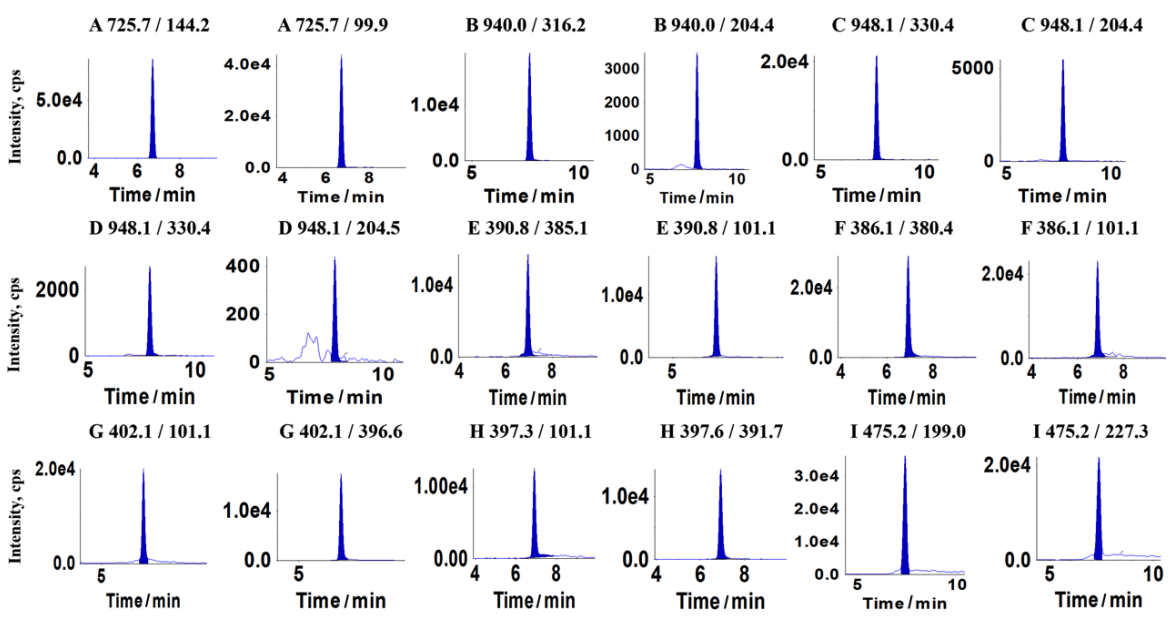

Fig. 4 Typical multi-reaction monitoring chromatograms of analytes in a lake water sample spiked at $0.2 \mu \mathrm{g} \mathrm{L}^{-1}$. A - I represent vancomycin, teicoplanin A2-1, teicoplanin A2-2\&A2-3, teicoplanin A2-4\&A2-5, polymyxin B1, polymyxin B2, colistin A, colistin B and bacitracin A, respectively.

water-soluble substances in lake water samples, leading to reduce impurities. Thus, $0.1 \% \mathrm{FA}$ in methanol-0.1\% FA in water $(80: 20, \mathrm{v} / \mathrm{v})$ was chosen as the reconstitution solution.

\section{Method performance}

Selectivity. For evaluating possible endogenous interferences, 50 blank lake water samples from different sources were analyzed by LC-MS/MS to validate the selectivity of the method. The result showed that no interference peaks appeared within the range of $\pm 2.5 \%$ of the retention time of all target drugs in the MRM chromatograms. Typical MRM chromatograms of blank lake water extract and blank lake water with 9 analytes spiked at $0.2 \mathrm{ng} \mathrm{mL}-1$ are illustrated in Figs. 3 and 4 . The target compounds and the impurity peak were well separated, proving that the method had good selectivity.

$\angle O D$ and $L O Q$. The LOD and LOQ were calculated in light of the method described above. As shown in Table 2, the LODs and LOQs of 9 peptide compounds in lake water samples ranged from $0.01-0.1$ and $0.02-0.2 n g \mathrm{~mL}^{-1}$, respectively. Since the pre-lyophilization method enriched the water sample by
50 times, the LODs and LOQs of this developed method were relatively low, indicating that the method was highly sensitive. Linearity. Within the experimental concentration range, the linearity of the chromatographic response was evaluated by the external standard method with a lake water matrix matching calibration curve. The linear range, linear equations and correlation coefficients are given in Table 2. The results show that the $r^{2}$ values of all analytes were higher than 0.99 , illustrating the matrix-matched standard calibration curves had a good linear relationship.

Recovery and precision. The recovery and RSD represented the accuracy and precision of the method. The recoveries of lake water at three concentrations are given. The results of the recoveries and RSDs are given in Table 3. The recoveries of all target compounds were above $70 \%(72.4-105.2 \%)$, except for teicoplanin A2-4\&A2-5 (65.8-75.1\%); the RSDs of intra-day and inter-day were less than $20 \%$, indicating that this developed method could meet the requirements of polypeptides residue analysis and detection. 
Table 2 Linearity, limit of detection (LOD) and limit of quantication (LOQ) of the developed method for target analytes in lake water

\begin{tabular}{llcrcc}
\hline \multicolumn{1}{c}{ Analyte } & \multicolumn{1}{c}{ Calibration curve } & $\begin{array}{c}\text { Correlation } \\
\text { coefficient, } r^{2}\end{array}$ & $\begin{array}{c}\text { Linear range/ } \\
\mathrm{ng} \mathrm{mL} \mathrm{mL}^{-1}\end{array}$ & $\begin{array}{c}\text { LOD/ } \\
\mathrm{ng} \mathrm{mL}\end{array}$ & $\begin{array}{c}\text { LOQ/ } \\
\mathrm{ng} \mathrm{mL}^{-1}\end{array}$ \\
\hline Vancomycin & $y=4.64 \times 10^{4} x+8.7 \times 10^{4}$ & 0.9980 & $1-200$ & 0.01 & 0.02 \\
Teicoplain A2-1 & $y=2.02 \times 10^{4} x+4.13 \times 10^{4}$ & 0.9987 & $10-300$ & 0.1 & 0.2 \\
Teicoplain A2-2\&A2-3 & $y=2 \times 10^{3} x+1.44 \times 10^{3}$ & 0.9915 & $10-300$ & 0.1 & 0.2 \\
Teicoplain A2-4\&A2-5 & $y=1.08 \times 10^{3} x+1.65 \times 10^{3}$ & 0.9973 & $10-300$ & 0.1 & 0.2 \\
Polymyxin B1 & $y=9.35 \times 10^{3} x+7.72 \times 10^{4}$ & 0.9941 & $10-300$ & 0.1 & 0.2 \\
Polymyxin B2 & $y=8.25 \times 10^{3} x+7.78 \times 10^{3}$ & 0.9954 & $5-200$ & 0.04 & 0.1 \\
Colistin A & $y=9.6 \times 10^{3} x+3.52 \times 10^{4}$ & 0.9975 & $5-200$ & 0.04 & 0.1 \\
Colistin B & $y=1.9 \times 10^{4} x+7.43 \times 10^{4}$ & 0.9931 & $5-200$ & 0.04 & 0.1 \\
Bacitracin A & $y=2.02 \times 10^{4} x+4.13 \times 10^{4}$ & 0.9980 & $10-300$ & 0.04 & 0.1 \\
\hline
\end{tabular}

Table 3 Recoveries and relative standard deviation (RSD) of target analytes in lake water

\begin{tabular}{|c|c|c|c|c|c|}
\hline \multirow{2}{*}{$\begin{array}{r}\text { Analyte } \\
\text { Vancomycin }\end{array}$} & \multirow{2}{*}{$\frac{\text { Spiked level/ng mL } \mathrm{mL}^{-1}}{0.04}$} & \multicolumn{3}{|c|}{ Intra-day recovery $(\mathrm{RSD}, \%, n=5)$} & \multirow{2}{*}{$\frac{\text { Inter-day recovery }(\mathrm{RSD}, \%, n=15)}{87.2(10.8)}$} \\
\hline & & $91.2(15.1)$ & $83.0(2.1)$ & $87.2(12.2)$ & \\
\hline & 0.1 & $87.2(14.4)$ & $83.3(5.5)$ & $80.4(17.8)$ & $83.6(12.2)$ \\
\hline & 0.2 & $97.3(9.3)$ & $96.3(13.4)$ & $92.0(7.6)$ & $95.2(9.4)$ \\
\hline \multirow[t]{3}{*}{ Teicoplain A2-1 } & 0.2 & $72.8(9.7)$ & $75.5(6.2)$ & $76.0(13.9)$ & $74.8(9.3)$ \\
\hline & 0.4 & $82.6(12.6)$ & $76.8(5.9)$ & $75.4(14.9)$ & $78.3(11.0)$ \\
\hline & 1 & $84.0(2.8)$ & $78.3(10.6)$ & $79.3(6.4)$ & $80.5(7.1)$ \\
\hline \multirow[t]{3}{*}{ Teicoplain A2-2\&A2-3 } & 0.2 & $72.4(8.9)$ & $74.9(14.1)$ & $80.1(13.9)$ & $75.8(11.9)$ \\
\hline & 0.4 & $79.0(10.4)$ & $76.5(7.0)$ & $76.3(10.1)$ & $77.3(8.2)$ \\
\hline & 1 & $82.6(8.9)$ & $82.0(5.3)$ & $76.8(11.8)$ & $80.5(8.5)$ \\
\hline \multirow[t]{3}{*}{ Teicoplain A2-4\&A2-5 } & 0.2 & $65.8(9.3)$ & $65.9(1.5)$ & $66.9(15.2)$ & $66.2(9.0)$ \\
\hline & 0.4 & $69.7(12.0)$ & $70.1(9.8)$ & $66.7(8.9)$ & $68.8(9.3)$ \\
\hline & 1 & $75.1(7.8)$ & $75.1(11.9)$ & $67.5(7.6)$ & $72.5(9.7)$ \\
\hline \multirow[t]{3}{*}{ Polymyxin B1 } & 0.2 & $84.6(12.9)$ & $90.3(9.4)$ & $92.0(3.5)$ & $89.0(8.8)$ \\
\hline & 0.4 & $90.8(9.8)$ & $87.0(5.1)$ & $81.9(11.2)$ & $86.6(9.0)$ \\
\hline & 1 & $91.2(6.8)$ & $90.5(6.8)$ & $88.7(2.4)$ & $90.1(5.1)$ \\
\hline \multirow[t]{3}{*}{ Polymyxin B2 } & 0.2 & $78.2(6.6)$ & $83.5(7.6)$ & $81.8(2.7)$ & $81.4(6.0)$ \\
\hline & 0.4 & $87.9(6.6)$ & $75.4(4.5)$ & $91.9(5.6)$ & $84.3(10.2)$ \\
\hline & 1 & $100.0(1.9)$ & $94.8(4.8)$ & $98.8(5.8)$ & $97.0(4.6)$ \\
\hline \multirow[t]{3}{*}{ Colistin A } & 0.2 & $101.7(17.9)$ & $84.7(7.4)$ & $81.2(4.3)$ & $89.8(15.9)$ \\
\hline & 0.4 & $87.9(5.1)$ & $75.8(5.1)$ & $89.6(9.8)$ & $84.4(10.0)$ \\
\hline & 1 & $100.0(8.6)$ & $99.1(0.9)$ & $102.3(1.5)$ & $100.5(4.6)$ \\
\hline \multirow[t]{3}{*}{ Colistin B } & 0.2 & $99.1(9.6)$ & 79.7 (6.1) & 83.7 (9.9) & $88.5(12.8)$ \\
\hline & 0.4 & 87.5 (11.6) & 77.9 (7.1) & $87.6(16.6)$ & 84.4 (12.4) \\
\hline & 1 & $105.2(4.6)$ & $96.4(8.2)$ & $96.4(5.1)$ & $99.3(6.9)$ \\
\hline \multirow[t]{3}{*}{ Bacitracin A } & 0.2 & $72.6(6.4)$ & 74.4 (4.4) & $79.6(12.1)$ & $75.6(8.5)$ \\
\hline & 0.4 & 73.5 (4.1) & $76.8(4.3)$ & 74.9 (12.7) & $75.0(7.3)$ \\
\hline & 1 & $85.4(5.0)$ & $83.2(11.6)$ & $83.1(6.8)$ & 83.9 (7.3) \\
\hline
\end{tabular}

\section{Matrix effects}

The results of ME for polypeptide antibiotics in lake water are illustrated in Fig. 5. Except for vancomycin, the mass detector signals for other polypeptide antibiotics were suppressed into different degrees. The ME for polymyxin B1 in lake water was weak, while other polypeptide antibiotics showed medium and strong suppression $(25.3-56.7 \%)$ in the matrix of lake water. In order to reduce quantitative deviations and to improve the accuracy of the determination results, the matrix matching standard curves were used for quantitative calibration, which could effectively compensate for the influence of the sample MEs on peptide antibiotics.

\section{Conclusions}

In this study, a sensitive, environmentally friendly and simple lyophilization pretreatment method was established for the simultaneous determination of 9 polypeptides in lake water matrices using LC-MS/MS. This method had good selectivity, linearity, accuracy and precision as well as lower LODs and LOQs. The developed method was appropriate for monitoring polypeptides antibiotics residues in lake water.

\section{Acknowledgements}

The authors are grateful to the financial support from the National Science Foundation of China (31572562) and the Key Program of Guangzhou Science and Technology Plan (201804020019).

\section{References}

1. W. Witte, Int. J. Antimicrob. Agents, 2000, 16, S19. 


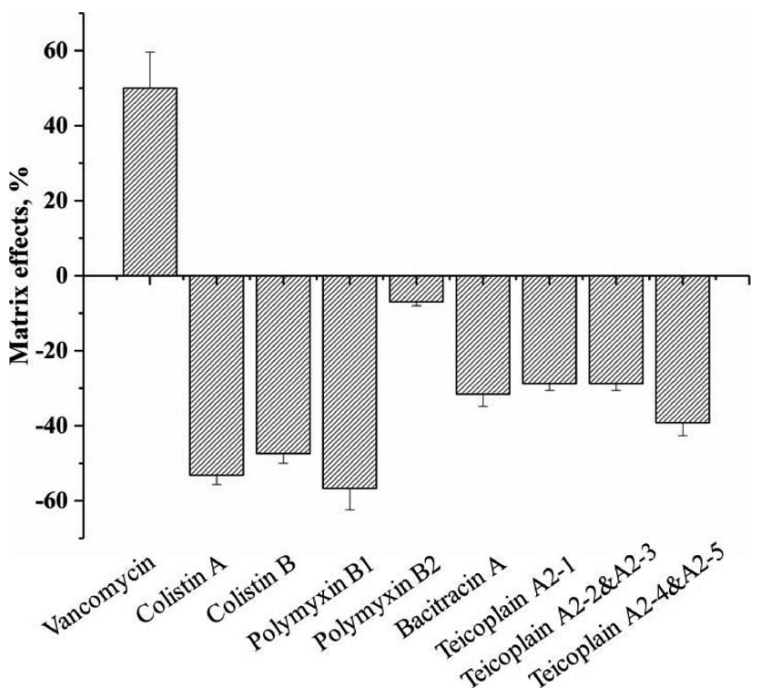

Fig. 5 Matrix effects of the lake water matrix on the response of target analytes.

2. J. O. Boison, S. Lee, and J. Matus, Anal. Bioanal. Chem., 2015, 407, 4065 .

3. X. Q. Song, J. M. Xie, M. Y. Zhang, Y. X. Zhang, J. F. Li, Q. W. Huang, and L. M. He, J. Chromatogr. B, 2018, 1076, 103.

4. L. Dijkshoorn, A. Nemec, and H. Seifert, Nat. Rev. Microbiol., 2007, 5, 939.

5. H. Giamarellou, Int. J. Antimicrob. Agents, 2010, 36, S50.

6. Communities C. Council regulation 2788/98, Official Journal of the European Communities Legislation, 1998.

7. Bulletin 194, Ministry of Agriculture, People's Republic of China, 2019.

8. Bulletin 235, Ministry of Agriculture, People's Republic of China, 2002.

9. CFR-Code of Federal Regulations Title 21, Food and Drug Administration of United States, 2019.

10. P. K. Jjemba, Agr. Ecosyst. Environ., 2002, 93, 267.

11. J. Li, R. W. Milne, R. L. Nation, J. D. Turnidge, T. C. Smeaton, and K. Coulthard, Antimicrob. Agents Chemother,
2003, 47, 1766.

12. A. K. Sarmah, M. T. Meyer, and A. B. A. Boxall, Chemosphere, 2006, 65, 725.

13. L. F. Du and W. K. Liu, Agron. Sustain. Dev., 2012, 32, 309.

14. Q. Qin, X. J. Chen, and J. Zhuang, Crit. Rev. Environ. Sci. Technol., 2015, 45, 1379.

15. I. T. Carvalho and L. Santos, Environ. Int., 2016, 94, 736.

16. Q. Fu, X. W. Li, K. N. Zheng, Y. B. Ke, Y. Y. Wang, L. N. Wang, F. G. Yu, and X. Xia, Food Chem., 2018, 248, 166.

17. X. Q. Song, Q. W. Huang, Y. X. Zhang, M. Y. Zhang, J. M. Xie, and L. M. He, J. Pharm. Biomed. Anal., 2019, 170, 234.

18. M. J. D. J. Valle, F. G. López, and A. S. Navarro, J. Pharm. Biomed. Anal., 2008, 48, 835.

19. S. M. Mũnoz and M. D. L. D. Castro, J. Chromatogr. A, 2005, $1066,1$.

20. X. Q. Song, E. Turiel, L. M. He, and A. M. Esteban, Polymers, 2020, 12, 594.

21. B. Li, T. Zhang, Z. Xu, and H. H. P. Fang, Anal. Chim. Acta, 2009, 645, 64.

22. J. Rossmanna, S. Schubert, R. Gurke, R. Oertel, and W. Kirch, J. Chromatogr. B, 2014, 969, 162.

23. K. A. Gaidhani, M. Harwalkar, D. Bhambere, and P. S. Nirgude, WJPR, 2015, 4, 516.

24. G. Kakiyama, A. Muto, H. Takei, H. Nittono, T. Murai, T. Kurosawa, A. F. Hofmann, W. M. Pandak, and J. S. Bajaj, J. Lipid. Res., 2014, 55, 978.

25. F. Y. Hu, K. Bian, Y. H. Liu, Y. J. Su, T. Zhou, X. Q. Song, and L. M. He, J. Chromatogr. A, 2014, 1368, 52.

26. F. Y. Hu, L. M. He, J. W. Yang, K. Bian, Z. N. Wang, H. C. Yang, and Y. H. Liu, J. Chromatogr. B, 2014, 949, 79.

27. R. Hirsch, T. A. Ternes, K. Haberer, A. Mehlich, F. Ballwanz, and K. L. Kratz, J. Chromatogr. A, 1998, 815, 213.

28. J. L. Qiu, Q. Y. Liu, M. Y. Zhang, X. Z. Li, J. H. Zhang, R. P. Xiong, and L. M. He, Anal. Lett., 2020, 1.

29. E. C. H. Wan, C. Ho, D. W. M. Sin, and Y. C. Wong, Anal. Bioanal. Chem., 2006, 385, 181.

30. J. A. Orwa, C. Govaerts, K. Gevers, E. Roets, A. V. Schepdael, and J. Hoogmartens, J. Pharm. Biomed. Anal., 2002, 29, 203. 